\title{
Inhomogeneous D-Wave Superconductivity and Antiferromagnetism in a Two-Dimensional Extended Hubbard Model with Nearest-Neighbor Attractive Interaction
}

\author{
W. P. Su \\ Department of Physics and Texas Center for Superconductivity, University of Houston, Houston, \\ Texas 77204, USA
}

\begin{abstract}
To understand the interplay of d-wave superconductivity and antiferromagnetism, we consider a two-dimensional extended Hubbard model with nearest neighbor attractive interaction. The Hamiltonian is solved in the mean-field approximation on a finite lattice. In the impurity-free case, the minimum energy solutions show phase separation as predicted previously based on free energy argument. The phase separation tendency implies that the system can be easily rendered inhomogeneous by a small external perturbation. Explicit solutions of a model including weak impurity potentials are indeed inhomogeneous in the spin-density-wave and d-wave pairing order parameters. Relevance of the results to the inhomogeneous cuprate superconductors is discussed.
\end{abstract}

PACS numbers: 74.20.-z, 74.72.-h, 74.25.Ha

Typeset using REVTEX 


\section{Introduction}

Motivated by the inhomogeneous $d$-wave superconductivity ${ }^{1-4}$ and antiferromagnetism ${ }^{5,6}$ observed in the cuprates, we have studied a two-dimensional extended Hubbard model with nearest-neighbor attractive interaction in a previous paper ${ }^{7}$ (hereafter referred to as I). Free energy of the homogeneous coexisting antiferromagnetic and superconducting state calculated as a function of band filling shows a region of negative curvature characteristic of a phase separation system ${ }^{8}$. In the two-phase region, the zero compressibility means that large fluctuations in density can be easily induced by small perturbations. Thus the potentials due to the randomly distributed dopants (impurities) could lead to inhomogeneous d-wave superconductivity and antiferromagnetism. In this paper, we solve the model Hamiltonian on a finite lattice in the mean-field approximation. Inhomogeneous solutions are indeed obtained as predicted in I.

This paper is organized as follows. First the model Hamiltonian (the tUVW model) is defined and the mean-field methodology is described in Section II. Solutions in the dopant free case are examined in Section III. Inhomogeneous solutions induced by random dopant potentials are presented in Section IV. Implications of the solutions are discussed in Section V.

\section{The tUVW Model}

The tUVW model ${ }^{9}$ is defined as

$$
\begin{aligned}
H=-t \sum_{<i j>\sigma}\left[c_{i \sigma}^{\dagger} c_{j \sigma}+H . c .\right]+U \sum_{i}\left(n_{i \uparrow}\right. & \left.-\frac{1}{2}\right)\left(n_{i \downarrow}-\frac{1}{2}\right)+V \sum_{<i j>}\left(n_{i}-1\right)\left(n_{j}-1\right) \\
& +\sum_{<i, j>^{\prime}} W_{i, j}\left(n_{i}-1\right)\left(n_{j}-1\right)-\mu \sum_{i} n_{i},
\end{aligned}
$$

where $n_{i \sigma}=c_{i \sigma}^{\dagger} c_{i \sigma}$ is a density operator of the conduction electrons, $n_{i}=n_{i \uparrow}+n_{i \downarrow}$, $<i j>$ is a nearest neighbor pair and $\langle i j\rangle^{\prime}$ is any other more distant pair. We measure energy in units of $t$ and set $t=1$. For homogeneous states, the extended repulsive terms 
enters only through $\mathrm{W}$, which is the summation of $W_{i j}$ over all extended pairs $<i j>^{\prime}$ divided by the total number of lattice sites. For inhomogeneous state calculations we need to specify the $W_{i j}$. To facilitate the calculations, we consider only next-nearest neighbor repulsive interactions. In Section IV the effect of a dopant is represented by a short range potential added to the Hamiltonian (1).

The tUVW model can be solved in the mean-field approximation by linearizing the interaction terms ${ }^{10,11}$. In linearizing the attractive $\mathrm{V}$ term, one arrives at three linear terms. The first is a density term, the second is a pairing term and the third is an exchange term. As opposed to most previous calculations, we retain all three terms. For the $\mathrm{W}$ term, only the density term is kept after linearization. The linear Hamiltonian is diagonalized by making a Bogoliubov transformation. As usual, self-consistent solutions are obtained by iterations. Spatially inhomogeneous solutions are readily obtained by starting from inhomogeneous initial configurations.

\section{Impurity Free Solutions}

All the calculations in this paper are done on a $24 \times 24$ lattice at zero temperature. To see the finite lattice effect and also to review the free energy argument for phase separation, we show in Figure 1 the free energy as a function of band filling on the finite lattice. The parameters used are the same as in I. Phase separation should occur between points A and B on the figure. There is essentially no difference between the finite lattice and infinite lattice results.

The parameters adopted in $\mathrm{I}(\mathrm{U}=2.1, \mathrm{~V}=-0.94, \mathrm{~W}=0.4)$ were chosen to reproduce the cuprate phase diagram. Empirically, we found that the $\mathrm{W}$ term is essential for this purpose. As an illustration, we show in Figure 2 the free energy curve for $\mathrm{U}=2.94, \mathrm{~V}=-0.9$ and $\mathrm{W}=0$. Phase separation still occurs, with a terminal bandfilling 0.25 , but the d-wave superconductivity (DSC) is confined to inside the two-phase region. It ceases to exist for band filling less than 0.275 . In contrast to the scenario presented in I, the phase separation boundary in this tUV model does not cut through the superconducting dome. In other words, homogeneous DSC does not exist in this case. 
As an example of phase separation in the tUVW model, we choose an average density (per spin) 0.4586 corresponding to 48 holes on the $24 \times 24$ lattice. In the simple Hubbard model, two widely spaced stripes ${ }^{12,13}$ (antiferromagnetic domain walls) are expected. In contrast, the solution of the tUVW model as depicted in Figure 3 is a narrow strip of low density (0.35) region embedded in a half-filled region. The half-filled region has the maximum magnetization(0.48), whereas the low density region has a nearly zero magnetization. The result thus is consistent with a phase separation scenario. By varying the band filling, we see similar results for band filling between A and B in Figure 1, i.e. the free energy argument for phase separation is explicitly demonstrated by finite lattice calculations.

In this connection, we have also examined a bipolaron solution corresponding to doping with two holes. As shown in Figure 4, there is a depression of the SDW order parameter (and the electron density) at the center of the bipolaron. This is expected because SDW is very stable at half-filling, any small change from half-filling is localized to a small region. What is surprising is the large local DSC order paramter present in the same neighborhood (Figure 5). This is a superconducting cloud surrounding a SDW bipolaron. For technical reasons, we can not obtain a polaron solution. We speculate that a polaron configuration would look qualitatively the same as a bipolaron. Assuming that to be true, the above result implies that although DSC is completely suppressed by SDW at half-filling in the ground state, the DSC phase does show up in the charged excited states (the polarons and bipolarons). This might explain the d-wave like dispersion of the photoexcited states in the undoped cuprates ${ }^{14}$.

\section{Inhomogeneous Solutions in the Presence of Impurities}

As we have alluded to in the Introduction, in the two-phase region the compressibility is zero because the free energy is a linear function of the density. That means an arbitrarily small perturbation can lead to significant density variation. In particular, even a very weak dopant or impurity potential can induce inhomogeneous solutions.

We model the impurity potential as follows: the potential at the impurity site is take to be $-0.35 t$, it reduces to $-0.175 t$ on the four nearest neighbors and vanishes elsewhere. We 
constrain the separation between two impurities to be larger than one lattice spacing. For a given distribution of impurities, the sum of the potentials is added as a diagonal term to the Hamiltonian (1). Only next-nearest neighbor pairs are considered for the repulsive W term.

For a typical case, we pick an average density of 0.417 per spin (96 holes). Sixty impurities are randomly positioned on the lattice as shown in Figure 6. Figure 7 depicts the variation of the DSC order parameter over the lattice. The average value of the DSC order parameter is 0.049. Obviously, there is a significant spatial variation (it varies from 0.01 to 0.073 ). The SDW order parameter depicted in Figure 8 clearly exhibits a high plateau region (half-filled region) and a low flat region (with zero magnetization). In addition, there is a region of negative magnetization. A very weak impurity potential (0.35t) seems to exert a strong influence in causing nanoscale inhomogeneities. It should be emphasized again that according to Figure 1, a homogeneous solution of this density (0.417) is superconducting but without SDW. Instead, phase separation together with weak impurity potential lead to nanoscale inhomogeneous superconductor. This provides the theoretical basis of an interpretation of the STM and ARPES data ${ }^{15-18}$ of the cuprates.

\section{Discussion}

The main point of this paper is to demonstrate that inhomogeneous DSC and SDW are natural consequences of the interplay between them. Both are derived from the UVW potential. Impurities alone without phase separation can also lead to inhomogeneous solutions as shown by other workers ${ }^{19-22}$. In our theory, the inhomogeneity is related to phase separation and therefore occurs within the two-phase region (at least for weak impurity potential).

It is useful to look at the results of this paper from a broader perspective. The attractive onsite Hubbard model has been employed as a standard model for studying an s-wave superconductor. The model can explain most of the superconducting properties while leaving the origin of the pairing force open. That could be mediated by phonons, excitons or something else. In a similar vein, the tUV model can be regarded as a standard model for a d-wave superconductor independent of the origin of the attractive pairing force (the 
attractive $\mathrm{V}$ term). Such a term could also generate s-wave superconductivity, therefore an onsite repulsive $\mathrm{U}$ term is needed to suppress it.

As is well known, the $\mathrm{U}$ term so introduced also leads to antiferromagnetism. The $\mathrm{V}$ term also has important implications other than generating DSC, it can cause phase separa$\operatorname{tion}^{7,10,23,24}$. The phase separation occurs at a temperature higher than the superconducting transition temperature. In that sense, it is more important than DSC. With the addition of an extended repulsive $\mathrm{W}$ term, the model can yield a phase diagram ${ }^{7}$ strikingly similar to the one observed in the cuprates ${ }^{25,26}$. In addition, inhomogeneous $\mathrm{DSC}^{4}$ is readily explained. From such a perspective, much of the mystery of the cuprate superconductors could simply be a consequence of $\mathrm{d}$-wave pairing in the appropriate parameter range, again independent of the origin of pairing.

In an s-wave superconductor, one can separate those effects which are due exclusively to electron-phonon coupling from those which are universally shared between all s-wave superconductors. The same thing should be done for a d-wave superconductor. It is pressing to figure out which portion of the cuprate phenomenology is d-wave universal and which portion is specific to pairing mechanism. That will help narrow the search for a pairing mechanism. The specific form of the interaction potential UVW, with its alternate signs, positive $\mathrm{U}$ and negative $\mathrm{V}$ plus positive $\mathrm{W}$ as the interparticle separation increases strongly resembles the Friedel oscillation. As such it might hint at a particular pairing mechanism.

This work was partially supported by the Texas Center for Superconductivity, the Robert A. Welch Foundation (grant number E-1070), and the National Science Council of Taiwan under contract number NSC 94-2811-M-110-005. I thank C. S. Ting for useful discussions. Thanks are also due to I. M. Jiang and the Department of Physics at the National Sun Yat-sen University for their hospitality during the summer of 2005. 


\section{REFERENCES}

${ }^{1}$ S.-H. Pan, J. P. O’Neal, R. L. Badzey, C. Chamon, H. Ding, J. R. Engelbrecht, Z. Wang, H. Eisaki, S. Uchida, A. K. Guptak, K.-W. Ng, E. W. Hudson, K. M. Lang, and J. C. Davis, Nature (London) 413, 282 (2001).

${ }^{2}$ C. Howald, P. Fournier, and A. Kapitulnik, Phys. Rev. B 64, 100504(R) (2001).

${ }^{3}$ K. M. Lang, V. Madhavan, J. E. Hoffman, E. W. Hudson, H. Eisaki, S. Uchida, and J. C. Davis, Nature (London) 415, 412 (2002).

${ }^{4}$ K. McElroy, D.-H. Lee, J. E. Hoffman, K. M. Lang, E. W. Hudson, H. Eisaki, S. Uchida, J. Lee, and J. C. Davis, cond-mat/0404005.

${ }^{5}$ S. Sanna, G. Allodi, G. Concas, A. D. Hillier, and R. De Renzi, Phys. Rev. Lett. bf 93, 207001(2004).

${ }^{6}$ Ch. Niedermayer, C. Bernhard, T. Blasius, A. Golnik, A. Moodenbaugh, and J. I. Budnick, Phys. Rev. Lett. 80, 3843(1998).

${ }^{7}$ W. P. Su, Mod. Phys. Lett. B 19, 1295(2005).

${ }^{8}$ Andrew Putnis, Introduction to Mineral Sciences, (Cambridge University Press, 1992).

${ }^{9}$ R. Micnas, J. Ranninger, S. Robaszkiewicz, and S. Tabor, Phys. Rev. B 37, 9410(1988).

${ }^{10}$ R. Micnas, J. Ranninger, and S. Robaszkiewicz, Phys. Rev. B 39, 11653(1989).

${ }^{11}$ R. Micnas, J. Ranninger, and S. Robaszkiewicz, Rev. Mod. Phys. 62, 113(1990).

12 J. Zaanen and O. Gunnarsson, Phys. Rev. B 40, R7391(1989).

${ }^{13}$ I. Martin, G. Ortiz, A. V. Balatsky, and A. R. Bishop, Europhys. Lett. 56, 845(2001).

${ }^{14}$ F. Ronning, C. Kim, D. L. Feng, D. S. Marshall, A. G. Loeser, L. L. Miller, J. N. Eckstein, I. Bozovic, and Z. X. Shen, Science 282, 2067(1998)

${ }^{15}$ L. Dell'Anna, J. Lorenzana, M. Capone, C. Castellani, and M. Grilli, Phys. Rev. B 71, 
064518(2005).

${ }^{16}$ Ming Cheng and W. P. Su, Phys. Rev. B 72, 094512(2005).

${ }^{17}$ A. C. Fang, L. Capriotti, D. J. Scalapino, S. A. Kivelson, N. Kaneko, M Greven, and A. Kapitulnik, Phys. Rev. Lett. 96, 017007(2006).

${ }^{18}$ Tamara S. Nunner, Wei Chen, Brian M. Andersen, Ashot Melikyan, and P. J. Hirschfeld, cond-mat/0509139.

${ }^{19}$ Ziqiang Wang, Jan R. Engelbrecht, Shancai Wang, Hong Ding, and Shuheng H. Pan, Phys. Rev. B 65, 064509(2002).

${ }^{20}$ Gonzalo Alvarez, Matthias Mayr, Adriana Moreo, and Elbio Dagotto, Phys. Rev. B 71, $014514(2005)$.

${ }^{21}$ W. A. Atkinson, Phys. Rev. B 71, 024516(2005).

${ }^{22}$ Tamara S. Nunner, Brian M. Andersen, Ashot Melikyan, and P. J. Hirschfeld, Phys. Rev. Lett. 95, 177003(2005).

${ }^{23}$ J. Wang, D. Y. Xing, J. Dong, and P. Hor, Phys. Rev. B 62, 9827(2000).

${ }^{24}$ E. V. L. de Mello and E. S. Caixeiro, Phys. Rev. B 70, 224517(2004).

${ }^{25}$ Tom Timusk and Bryan Statt, Rep. Prog. Phys. 62, 61(1999).

${ }^{26}$ J. L. Tallon and J. W. Loran, Physica C 349, 53(2001). 


\section{FIGURES}

Figure 1. Free energy (per site) of the homogeneous d-wave superconducting state (squares) and that of the homogeneous coexisting SDW and DSC state (circles) at zero temperature in the tUVW model.

Figure 2. Same as Figure 1 for the tUV model.

Figure 3. Charge density profile of a solution of the tUVW model corresponding to an average density of 0.4586 per spin. The strip of low density (0.35) region extends in the forward direction. The plane at the bottom is the lattice plane, the charge density corresponds to the height of the surface above this plane.

Figure 4. Spin-density-wave profile of a bipolaron.

Figure 5. D-wave superconducting order parameter profile of a bipolaron. The order param-

eter is defined as $\Delta_{d}=\left|\frac{V}{2}<c_{i \uparrow} c_{j \downarrow}-c_{i \downarrow} c_{j \uparrow}>\right|$ (i,j is a nearest neighbor pair) The maximun of $\Delta$ at the center of the bipolaron is 0.064 .

Figure 6. Positions of sixty impurities randomly distributed on the lattice.

Figure 7. Spatial variation of the DSC order parameter of an inhomogeneous solution with average density 0.416 per spin in the presence of sixty impurites shown in Figure 6 .

Figure 8. Spatial variation of the SDW order parameter over the lattice plane in the inhomogeneous solution described in Figure 7. 
This figure "fig1.jpg" is available in "jpg" format from: http://arxiv.org/ps/cond-mat/0604400v1 
This figure "fig2.jpg" is available in "jpg" format from: http://arxiv.org/ps/cond-mat/0604400v1 
This figure "fig3.jpg" is available in "jpg" format from: http://arxiv.org/ps/cond-mat/0604400v1 
This figure "fig4.jpg" is available in "jpg" format from: http://arxiv.org/ps/cond-mat/0604400v1 
This figure "fig5.jpg" is available in "jpg" format from: http://arxiv.org/ps/cond-mat/0604400v1 
This figure "fig6.jpg" is available in "jpg" format from: http://arxiv.org/ps/cond-mat/0604400v1 
This figure "fig7.jpg" is available in "jpg" format from: http://arxiv.org/ps/cond-mat/0604400v1 
This figure "fig8.jpg" is available in "jpg" format from: http://arxiv.org/ps/cond-mat/0604400v1 